\title{
Les thèses en environnement industriel Le cas du groupe THALES
}

Jean-Claude Boudenot (jean-claude.boudenot@thalesgroup.com) Thales Research and Technology, Route départementale 128, 91767 Palaiseau

\section{Les thèses}

\section{en environnement}

industriel représentent

non seulement une

excellente formation,

mais assurent un lien

précieux entre les uni-

versités, les centres de

recherches académiques

et les entreprises.

\section{Elles contribuent à}

accélérer le processus de transfert de l'innovation vers la réalisation.

Comme le rappelle

souvent Albert Fert

(UMR CNRS-Thales, prix

Nobel de physique 2007),

c'est souvent au moment

de la thèse qu'apparaissent

des idées qui peuvent

se développer pendant

une partie de la future

carrière.

\section{Nous indiquons ici}

l'importance des thèses dans un groupe industriel comme Thales, soulignée par les témoignages de quelques doctorants et jeunes embauchés.

(1) CIFRE : Convention Industrielle de Formation par la Recherche.

(2) ANRT : Association Nationale de la Recherche Technique.

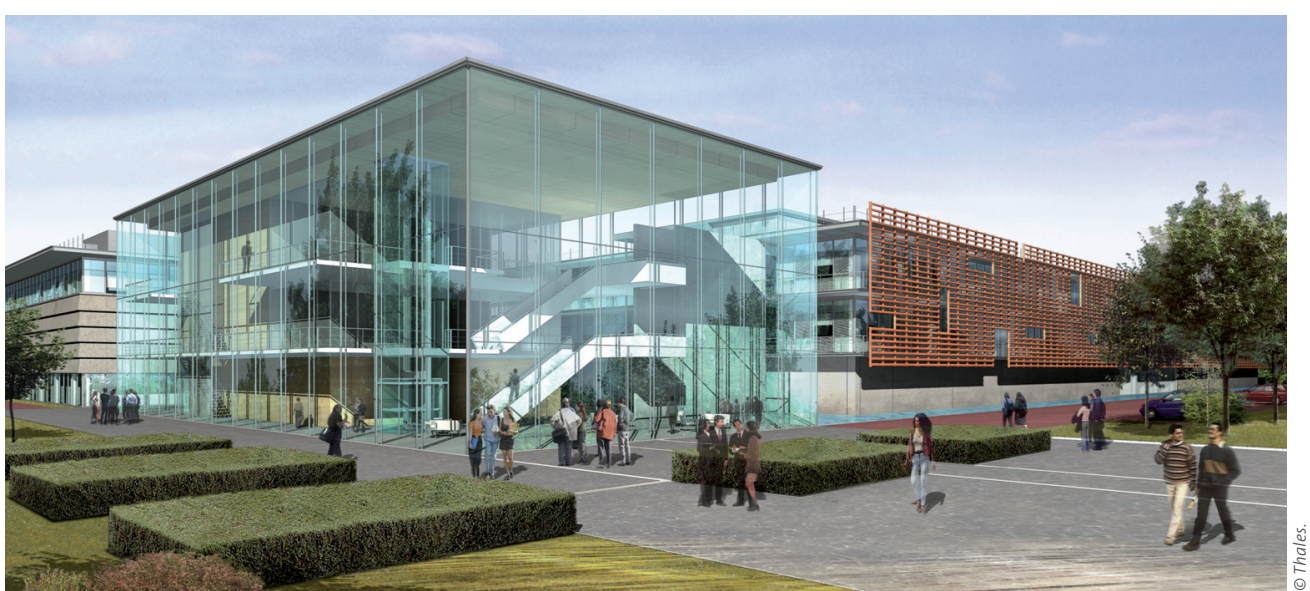

Le bâtiment de TRT (Thales Research and Technology) sur le site de l'École polytechnique, à Palaiseau.

Dans le cadre du forum des stagiaires de Thales (le groupe accueille environ 2000 stagiaires par an) qui s'est déroulé le 24 mai 2007, une table ronde sur le thème «Les thèses en environnement industriel " a été organisée de façon à faire mieux connaitre aux étudiants cette possibilité de formation par la recherche et les débouchés offerts dans le monde industriel. En France, le groupe Thales (hors activités spatiales) compte environ 150 doctorants, répartis à 40\% dans le centre de recherche de Palaiseau et à 60\% dans les divisions opérationnelles (voir encadré p. 22). La table ronde réunissait Martin Defour, Directeur technique de la division aéronautique (l'une des six divisions de Thales) ; Philippe Gautier, responsable des thèses $\operatorname{CIFRE}^{(1)}$ à l'ANRT $^{(2)}$; trois jeunes embauchés ayant fait leur thèse au sein de Thales : Sylvain Schwartz, Sébastien Demoustier et Éric Minoux, ainsi que trois doctorants : Michaël Soulignac, Ludovic Schreider et David Monnereau.

"Notre division aéronautique, a souligné Martin Defour, s'est donnée un double objectif: le premier est d'augmenter de façon significative le nombre de doctorants, avec une cible visée de 30 à 35 doctorants par an. Le second objectif est d'embaucher ces doctorants après leur thèse, avec un taux de plus $50 \%$. " (Ce taux, essentiellement lié à la qualité des travaux effectués et des qualités humaines des candidats, pourrait être plus important.) « La formation par la recherche est une excellente école, ajoute Martin Defour, et à l'issue des trois ans de thèse, le doctorant connaît bien l'environnement de la division et nous le connaissons également bien, ce qui est un facteur très favorable à l'embauche. Les travaux de recherche effectués au cours de la thèse sont, de plus, une excellente opportunité d'établir des contacts solides entre notre monde industriel et les laboratoires académiques. En moyenne, nos doctorants passent $60 \%$ de leur temps au sein du laboratoire académique et $40 \%$ de leur temps au sein de l'entreprise. Cette répartition n'est pas figée : selon les circonstances (thèmes traités, capacité d'encadrement), les doctorants peuvent passer plus de temps dans le laboratoire universitaire, ou inversement dans l'entreprise. "

Philippe Gautier a, quant à lui, rappelé l'importance du dispositif CIFRE. Les thèses CIFRE ont été lancées en 1981. Après plus de 25 ans d'existence, ce dispositif a permis à plus de 15000 doctorants d'être formés par la recherche dans un milieu industriel. « La convention est signée, rappelle Philippe Gautier, par les trois partenaires : le chercheur, l'entreprise qui lui confie un projet, et le laboratoire d'accueil. La convention CIFRE s'applique également aux doctorants d'origine étrangère (16\% des cas en 2005) ; la seule condition est que l'entreprise d'accueil soit de droit français. L'État a prévu de consacrer $10 \%$ de moyens supplémentaires chaque année d'ici 2010, avec l'ambitieux objectif de signer à cette échéance 2000 conventions CIFRE par an.»

Dans leurs témoignages, qui sont rapportés ci-après, les jeunes participants à la table ronde ont rappelé brièvement leur parcours et exprimé leur point de vue sur la thèse en milieu industriel. 


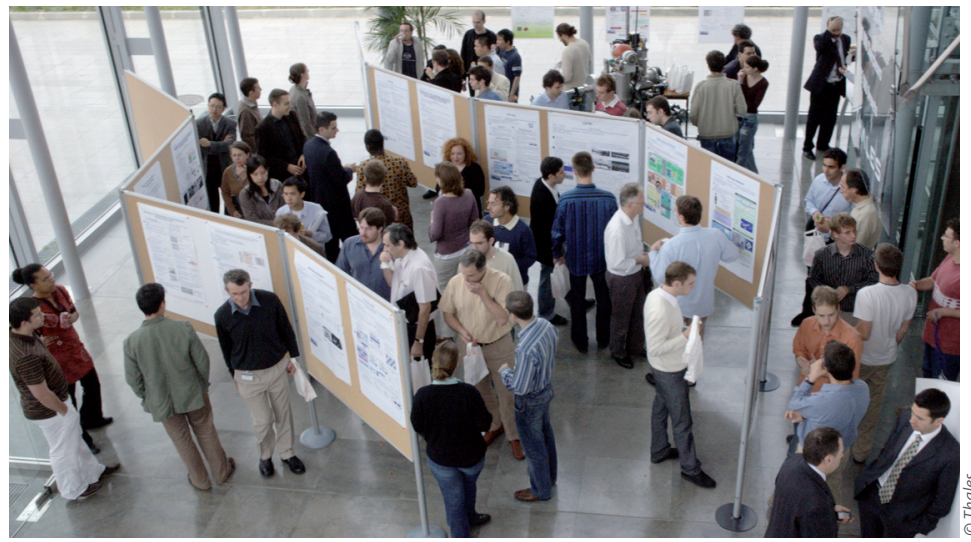

\section{Les thèses dans le groupe Thales en France}

Répartition par domaine

Micro-ondes, Optoélectronique, Optronique

Traitement de l'information et fusion de données

Ingénierie des systèmes complexes.

Architecture informatique .

Technologies pour la sécurité et applications.

Modes de financement

Thèses CIFRE

Thèses DGA

Thèses BDI, ONERA, ministère de la Recherche ..

Journée de thèse à Thales Research and Technology: comme chaque année, les doctorants de TRT présentent leurs travaux aux chercheurs du centre de recherche de Palaiseau.

\section{Une thèse récompensée}

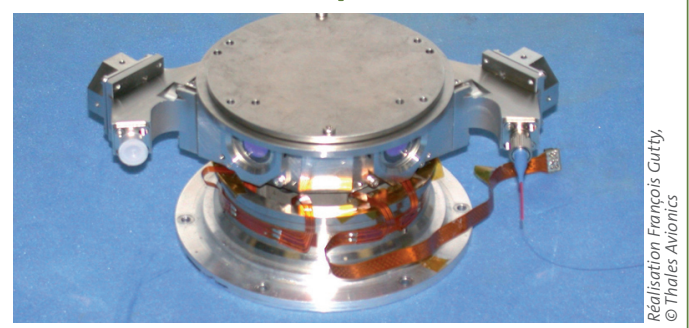

Prototype du gyrolaser en anneau à état solide.

Sylvain Schwartz est diplômé de l’École polytechnique et de l'École nationale supérieure des télécommunications. Il a effectué ses travaux de doctorat à TRT et y est maintenant ingénieur de recherche. Ces travaux de thèse ont porté sur l'étude d'un nouveau type de gyromètre optique, le " gyrolaser à état solide ". II s'agit d'un instrument utilisant certaines propriétés des lasers en anneau pour mesurer des rotations, ce qui permet par exemple de guider un avion ou une fusée sans l'aide de références externes comme le GPS. Ces travaux ont été effectués en partenariat étroit avec Thales Aerospace, et ont débouché sur la fabrication d'un prototype, première étape vers un produit industriel.

Ces travaux ont donné lieu à plusieurs publications scientifiques, ainsi qu'au dépôt d'une dizaine de brevets. Ils ont été couronnés par l'un des douze prix de thèse de l'École polytechnique 2007, ainsi que par le deuxième Prix de l'innovation technologique Air et espace du salon du Bourget 2007.

\section{Le témoignage de Sylvain Schwartz (Ingénieur de recherche).}

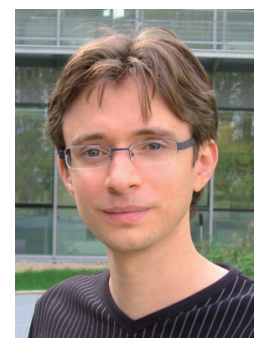

La thèse représente l'occasion unique, pour qui est appelé à travailler par la suite dans l'industrie, de se consacrer trois pleines années durant à un projet innovant, ce qui permet d'aller au fond des choses, de se mettre au niveau de l'état de l'art mondial, de réaliser des travaux originaux et d'apporter sa pierre à "l'édifice de la science ». Par ailleurs, le doctorant développe au cours de ces trois années, en plus de ses connaissances techniques, des compétences et un savoir-faire très utiles en milieu industriel : savoir respecter des objectifs sous contraintes de budget et de temps, gérer un projet, travailler en équipe, traiter avec des fournisseurs... Si la thèse est, de plus, effectuée directement en milieu industriel, elle permet d'acquérir une connaissance de l'entreprise, ce qui est un atout majeur pour la suite. Parmi les multiples possibilités qui sont offertes au doctorant, Thales Research and Technology (TRT), qui est le laboratoire de recherche du groupe Thales, présente l'avantage d'une grande proximité à la fois avec les unités opérationnelles du groupe et avec le monde académique alentour, notamment l'École polytechnique et l'Institut d'Optique Graduate School. Environnement scientifique de très haut niveau en phase avec les besoins d'innovation d'une entreprise essentiellement technologique, il constitue un cadre idéal pour des travaux de doctorat à vocation industrielle.

Le témoignage de Michaël Soulignac (Doctorant, 25 ans, formation : École d'ingénieur en informatique (EISTI, Cergy-Pontoise) avec en parallèle un DEA en informatique à l'université Paris XIII).

Faire une thèse en environnement industriel permet de travailler sur un thème de recherche ayant un potentiel d'application pour l'entreprise (personnellement, le sujet que je traite concerne la planification de trajectoire pour des drones en mission). Par ailleurs, cela donne la possibilité de découvrir la vie de l'entreprise et d'y participer, l'occasion de piloter un projet en s'y impliquant totalement, et d'accumuler une expérience de trois ans. Cette formation par la recherche permet de découvrir l'innovation et de porter un regard différent sur les problèmes. Elle conduit à l'acquisition de qualités telles que la capacité de recul ou la capacité d'analyse et de synthèse, très utiles pour l'entreprise. 


\section{Le témoignage de Sébastien Demoustier}

\section{(Ingénieur de recherche, 28 ans, formation Sup'Optique).}

Je suis entré dans le groupe Thales par le biais d'un stage à Singapour, qui fut une expérience des plus enrichissantes, tant du point de vue professionnel et humain, par la découverte et l'adaptation à une culture différente, qu'en termes d'orientation de carrière, puisque c'est en observant la coopération technologique de haut niveau entre nos deux pays que j'ai réalisé la valeur ajoutée que pouvait apporter une thèse en milieu industriel.

J'ai donc choisi de faire une thèse chez Thales, d'une part pour me forger une expertise solide dans les lasers à fibre optique, domaine en plein essor dont les applications vont de la défense au biomédical et, d'autre part, pour être confronté et résoudre de manière autonome le type de problème que pose la mise au point de technologies émergentes. Ainsi, il est clair qu'à l'issue de la thèse, j'avais acquis un savoir pointu dans mon domaine, mais aussi de nouvelles aptitudes dans ma façon de travailler : créativité, ouverture sur l'extérieur, transmission des connaissances, autonomie, capacité à réagir face à l'imprévu. Je souhaitais faire cette thèse en milieu industriel pour donner à mes travaux de recherche une perspective d'application potentielle à moyen terme et garder ainsi en tête, autant que possible, les notions de contraintes matérielles, de temps et de côtts.

Après la thèse, de nombreuses propositions m'ont été faites, tant en recherche que plus en aval dans le développement et l'industrialisation de produits, au sein de Thales et dans d'autres compagnies. J'ai choisi un poste d'ingénieur R\&D chez Thales dans le domaine des hyperfréquences, pour élargir mon spectre de compétences métier. J'ai également été séduit par le fait qu'on me demande aujourd'hui d'amener le fruit de la recherche vers la maturité requise par les innovations de demain.

\section{Le témoignage de David Monnereau}

\section{(Doctorant à Thales Communications, site de Colombes)}

Le choix de mon orientation vers une thèse en convention CIFRE n'était pas immédiat. Suite à un DUT, je suis entré à l'École polytechnique de l'université de Nantes qui proposait, en parallèle de la dernière année du cursus d'ingénieur, de suivre des cours de master de recherche. Des recherches personnelles et des discussions avec des enseignants m'ont permis de comprendre qu'une thèse, notamment par le biais d'une convention CIFRE, ne servait pas uniquement à devenir enseignant-chercheur. De mon point de vue, ce type de convention offre plusieurs avantages. Premièrement, elle permet d'être en relation avec un industriel cherchant à répondre à un de ses problèmes et encadrant la thèse. Ensuite, les trois années étant reconnues comme des années d'expériences et la rémunération étant de niveau correct (au minimum $20700 €$ en 2006), une CIFRE ne présente pas vraiment de désavantages par rapport à un poste d'ingénieur. D'autre part, une CIFRE permet d'obtenir le grade de docteur, mieux reconnu qu'un diplôme d'ingénieur au niveau international. Enfin, l'étude approfondie et en autonomie, pendant trois ans, d'un sujet portant sur une technologie de pointe permet d'obtenir une expertise dans ce domaine, d'apprendre à se connaître, à se gérer, ainsi qu'à gérer un projet, atouts très intéressants si l'on souhaite accéder à des postes de management par la suite.

Lors de ma recherche d'emploi,j'étudiais à la fois les offres de postes d'ingénieurs et les offres de thèses CIFRE. Je favorisais d'abord la qualité du sujet du poste ou de la thèse. Maintenant, je suis en thèse à Thales et je travaille dans le domaine des systèmes électroniques, avec une problématique portant à la fois sur du matériel et du logiciel, ce qui n'était pas évident à trouver au moment de ma recherche d'emploi. Le laboratoire de recherche avec lequel je travaille, le LESTER, étant à Lorient, et le matériel que j'étudie étant à Colombes, je passe la quasi-totalité de mon temps dans l'entreprise, qui offre un environnement propice à mon travail de recherche, particulièrement agréable et motivant.

\section{Le tếmoignage d'Eric Minoux (Ingénieur de recherche).}

À ma sortie de l'École supérieure d'optique, j'ai effectué une thèse sur les nanotubes de carbone entre Thales Research \& Technology (TRT) et le Laboratoire de physique des interfaces et des couches minces (LPICM) de l'École polytechnique. Cette thèse en milieu industriel (contrat CIFRE) a été l'occasion pour moi de travailler trois ans sur un sujet passionnant, de me forger une réelle expertise dans un domaine pointu, et d'acquérir une méthode de travail unique allant de la réflexion théorique aux expérimentations pratiques, et de la présentation de mon travail lors de conférences internationales à la gestion de projet. Cette première expérience m'a conforté dans mon choix de travailler ultérieurement en recherche appliquée, sur de nouvelles technologies innovantes avec applications industrielles.

Je travaille aujourd'hui entre TRT et Thales Systèmes Aéroportés (TAS) sur des micro/nano-systèmes électromécaniques (MEMS/NEMS), qui ouvrent de nouvelles fonctionnalités et applications dans le domaine des radars. Dans ce cadre, je suis amené à côtoyer aussi bien des universitaires, pour résoudre des problèmes scientifiques, que des industriels, pour mieux cerner les contraintes liées aux produits. Ces deux aspects constituent une expérience particulièrement enrichissante et formatrice, TRT apparaissant comme une passerelle entre les mondes académique et industriel. 